\title{
¿POR QUÉ SON TAN DIFÍCILES LOS PACTOS EDUCATIVOS?
}

\author{
Juan Carlos Tedesco (*)
}

SÍNTESIS: Si la sociedad surgida de la aceleración de los desarrollos científicos y tecnológicos encuentra su mejor concreción en la centralidad del conocimiento, parece lícito concluir que la educación debe ser uno de los factores que garanticen e impulsen el éxito de ese prototipo social.

Sin embargo, después de tres décadas de sociedad del conocimiento, los efectos no deseables de su implantación y expansión parecen estar propiciando la aparición de un nuevo tipo de inequidad, con consecuencias más devastadoras que las producidas por el modelo de producción industrial precedente.

Esta situación propone la necesidad de recuperar para la educación su papel de transmisora de valores de solidaridad, que fundamenten políticas de redistribución tendientes a construir una relación virtuosa entre conocimiento, solidaridad y equidad.

En este sentido, los consensos que sean posible establecer en torno al modelo educativo y su consecución, pueden favorecer la adopción de una forma socialmente significativa de toma de decisiones que consolide la ejecución de aquellas políticas.

No obstante, debe tenerse presente que los pactos educativos no pueden ser un fin en sí mismos. En todo caso, serán sólo instrumentos válidos en el contexto de un proyecto social basado en la idea de construir una sociedad equitativa y dinámica.

SÍNTESE: Se a sociedade surgida da aceleração dos desenvolvimentos científicos e tecnológicos encontra sua melhor concreção na centralidade do conhecimento, parece lícito concluir que a educação deve ser um dos fatores que garantem e impulsionem o êxito desse protótipo social.

No entanto, depois de três décadas de sociedade do conhecimento, os efeitos não desejáveis de sua implantação e expansão parecem estar propiciando a aparição de um novo tipo de ineqüidade, com conseqüências mais devastadoras que as produzidas pelo modelo de produção industrial precedente.

(*) Director de la sede en Buenos Aires del Instituto Internacional de Planeamiento de la Educación (IIPE) de la UNESCO. 
Esta situação propõe a necessidade de recuperar para a educação seu papel de transmissora de valores de solidariedade, que fundamentem políticas de redistribuição com tendências a construir uma relação virtuosa entre conhecimento, solidariedade e eqüidade.

Neste sentido, os consensos que sejam possíveis ser estabelecidos em torno do modelo educacional e sua consecução, podem favorecer a adoção de uma forma socialmente significativa de tomada de decisões que consolide a execução de tais políticas.

Não obstante, deve ter-se presente que os pactos educacionais não podem ser um fim em si mesmos. Serão somente instrumentos válidos no contexto de um projeto social baseado na idéia de construir uma sociedade eqüitativa e dinâmica.

Reconocer que las estrategias de acción educativa deben ser diseñadas a través de la participación de todos los actores sociales, es un lugar común en la reciente literatura sobre políticas educativas. La educación es un proceso muy complejo, donde la responsabilidad y la autoridad tienen que ser compartidas por los citados actores. A su vez, la continuidad en la aplicación de las estrategias de transformación ha sido reconocida como una de sus condiciones de éxito, y para que exista continuidad -al menos en contextos democráticos-, es necesaria la existencia de un nivel básico de acuerdo y de compromiso de esos actores en su aplicación. En tal sentido, la última década del siglo XX se inició con un clima de optimismo acerca de la existencia de condiciones favorables para la definición de estrategias educativas mediante la participación y el consenso de los diferentes actores. Ese optimismo se puso de manifiesto, por ejemplo, en la Conferencia Internacional Educación para Todos, realizada en Jomtiem, que fue convocada por los principales organismos de cooperación internacional, y en la que uno de sus postulados básicos fue la necesidad y la posibilidad de establecer nuevas alianzas en favor de la educación ${ }^{1}$. El factor clave del optimismo fue la constatación de que en los nuevos escenarios sociales que surgían a partir de la expansión de las nuevas tecnologías y de la democracia política, el conocimiento constituye la variable central, tanto desde el punto de vista de la competitividad económica como del desempeño ciudadano y de la equidad social.

Sin embargo, la realidad se encargó de demostrar que superar el mero reconocimiento retórico acerca de la necesidad de concertar las

${ }^{1}$ World Conference on Education for All (1990): Meeting Basic Learning Needs: A Mission for the 1990's. Background Document, Jomtien, Thailand, 5-9 march. 
políticas educativas implica enfrentar dificultades muy importantes, algunas específicas de los nuevos escenarios sociales. Por ello, es posible postular la hipótesis de que el origen de las dificultades para concertar políticas educativas radica en la propia centralidad que hoy ocupa el conocimiento en la estructura social. Para decirlo en pocas palabras, en la medida en que la información y el conocimiento constituyen cada vez más las variables claves de la distribución del poder, el control de su producción y de su distribución se convierten en el ámbito donde se desarrollan y se desarrollarán en el futuro los conflictos sociales más significativos ${ }^{2}$.

La evolución social reciente ha permitido apreciar que, en contra de los pronósticos de las hipótesis optimistas sobre las potencialidades democráticas de una economía y de una sociedad basada en la producción de conocimientos ${ }^{3}$, las economías productoras de ideas parecen ser más inequitativas que las que fabrican objetos. Tal como expresa Cohen, la propensión a excluir a los que no tienen ideas es más fuerte que la propensión a excluir a los que no tienen riquezas. La lógica que operaría en las economías productoras de conocimientos sería la de la calidad total o la del «error 0». En este tipo de funcionamiento, el menor error pone en crisis la cadena de producción, razón por la cual las calificaciones de los trabajadores en todos los puestos de trabajo deben ser muy altas. Con tal forma de funcionar, como sostiene Cohen, los mejores se juntan con los mejores y los mediocres con los mediocres. Las nuevas tecnologías exacerban esta tendencia más o menos natural, al favorecer la descentralización, la externalización de actividades y el achatamiento de las pirámides de organización jerárquica de las unidades de producción ${ }^{4}$.

En este nuevo escenario se modifican las tradicionales formas de segmentación propias del capitalismo industrial y del modelo fordista de organización del trabajo. De acuerdo con los estudios sobre el tema, la segmentación tiende ahora a establecerse entre bloques completos de unidades productivas, y no entre sectores de una determinada empresa o sector de producción. La miseria del capitalismo actual consiste en

\footnotetext{
${ }^{2}$ Véase Juan Carlos Tedesco (1995): El nuevo pacto educativo. Competitividad y ciudadanía en la sociedad moderna, Madrid, Alauda-Anaya.

${ }^{3}$ Una versión muy popular de estas hipótesis optimistas puede verse en Alvin Toffler (1990): El cambio del poder, Barcelona, Plaza y Janés.

${ }^{4}$ David Cohen (1998): Riqueza del mundo, pobreza de las naciones, Buenos Aires, Fondo de Cultura Económica.
} 
crear, en el seno de cada grupo social, tensiones que hasta ahora estaban en el ámbito de las rivalidades intergrupos ${ }^{5}$. La dinámica del proceso productivo explica la aparente paradoja que confronta la observación de los procesos sociales contemporáneos, donde se advierte que el nuevo modo de producción se caracteriza por crear más igualdad y más desigualdad al mismo tiempo. Entre los que se incorporan al proceso productivo tecnológicamente más avanzado, existe mucha más homogeneidad que en el pasado, pero entre ellos y el resto de los que se desempeñan en unidades productivas atrasadas o que son excluidos del proceso productivo, se establecen grandes distancias ${ }^{6}$.

El modelo de organización del trabajo que nos ocupa tiene consecuencias sobre la dinámica del empleo y de los salarios. No analizaremos aquí el fenómeno, pero hay consenso en reconocer que uno de los resultados más visibles de estos procesos es la concentración del ingreso y el aumento de la desigualdad. Ambos son factores muy importantes en América Latina, que se ha transformado en la región más inequitativa del mundo ${ }^{7}$. La discusión acerca del vínculo entre modernización tecnológica, globalización y desigualdad, es una de las discusiones más relevantes del momento ${ }^{8}$. Pero, más allá de las hipótesis que se puedan formular, lo cierto es que la concertación sobre las políticas educativas en un contexto de tal tipo no puede ser un proceso exento de dificultades y de conflictos. Concertar en un contexto en el que existe una fuerte tendencia a excluir y a expulsar exige poner en juego dimensiones distintas a las tradicionales. Desde esa perspectiva, apelar a la concertación de políticas educativas no es sólo postular una forma o una metodología de enfrentar el conflicto, sino que, en sí misma, implica un contenido

${ }^{5}$ D. Cohen, op. cit.

${ }^{6}$ La literatura al respecto es muy abundante. Puede verse el ya clásico libro de Manuel Castells La era de la información. Economía, sociedad y cultura (3 vols.), Madrid, Alianza, 1997. También, Robert Castel Metamorfosis de la cuestión social. Una crónica del salariado, Buenos Aires, Paidós, 1997. Un resumen desde la dimensión educativa puede verse en Juan Carlos Tedesco Educar en la sociedad del conocimiento, Buenos Aires, Fondo de Cultura Económica, 2002.

7 Según datos procesados por el PNUD, el coeficiente de Gini para el conjunto de la región creció entre 1990 y 2003 del 0,554 al 0,566, mientras que el promedio mundial es de 0,381.

8 Ver, por ejemplo, los trabajos incluidos en Fernando Calderón (coord.) ¿Es sostenible la globalización en América Latina?; Debates con Manuel Castells (2 vols.), Santiago de Chile, Fondo de Cultura Económica, 2003, especialmente el texto de A. Ortuño y C. Pinc «Globalización, desigualdad y reformas en la América Latina de los años noventa». 
socialmente significativo, ya que la forma como se toman decisiones educativas es hoy uno de los debates que divide posiciones e intereses antagónicos.

La experiencia reciente indica que la concertación es rechazada o resistida desde dos perspectivas: una es la que proviene de los enfoques económicos y políticos de inspiración neoliberal, según los cuales las decisiones educativas no pueden someterse a procesos de concertación ni de negociación política, sino que deben ser dejadas a los mecanismos del mercado como el resto de las decisiones sobre distribución de bienes y servicios, donde la lógica del comportamiento ciudadano es reemplazada por la del "cliente». La otra es la que proviene de los enfoques fundamentalistas autoritarios, según los cuales las decisiones son o deben ser tomadas sólo por los que controlan el manejo del aparato del Estado, excluyendo toda posibilidad de pluralismo y de debate.

En síntesis, la diferencia fundamental entre la concertación de políticas educativas y las alternativas del mercado, por un lado, o del fundamentalismo autoritario por el otro, radica en el papel que se asigne a la dimensión política. Apelar a la concertación implica resguardar la esfera de la política en la toma de decisiones, ya que obliga a cada actor social a discutir y a negociar públicamente sus opciones educativas. EI mercado, en cambio, suprime la política y deja la toma de decisiones librada al resultado de determinaciones individuales, en función de intereses y de posibilidades particulares y de corto plazo. El fundamentalismo autoritario, a su vez, elimina la política porque deja todo el poder en manos de un solo actor social. El gran interrogante que abre esta discusión consiste en saber si la política tiene o tendrá la fuerza suficiente como para contrarrestar el peso de las tendencias propias de los intereses económicos.

Destacar la relevancia de la dimensión política frente a las alternativas fundamentalistas de mercado o autoritarias, abre la discusión sobre las características de la política en el nuevo capitalismo en general, y en América Latina en particular. En ese sentido, la paradoja radica en que las mayores demandas que se presentan al comportamiento político están acompañadas por una importante erosión de las instituciones del Estado para satisfacer las demandas sociales y las de los partidos políticos como organizaciones representativas de la ciudadanía. Desde un punto de vista general, el proceso de globalización ha puesto en crisis las instituciones del Estado-nación, y ha provocado la pérdida de capacidad de control democrático sobre un conjunto de decisiones 
políticas. Ulrich Beck ha analizado la dinámica del poder mundial y ha Ilamado la atención sobre el déficit de institucionalidad política capaz de enfrentar los fenóm enos de la m undialización ${ }^{9}$. Toda la institucionalidad política está basada en la idea del Estado-nación, y sólo de forma muy precaria aparecen mecanismos institucionales capaces de articular discusiones de carácter mundial. Eso implica que muchas decisiones se adoptan sin discusión y sin procesos de concertación real, porque no existen dispositivos políticos capaces de garantizar procesos democráticos en la toma de decisiones fuera del ámbito del Estado-nación. Los campos donde se pone de manifiesto este déficit institucional de manera más visible son los económico-financieros y los vinculados con riesgos globales tales como el cuidado del medio ambiente, el terrorismo internacional, el narcotráfico, etc. Sin embargo, cada vez más aparecen decisiones de impacto educativo y cultural que no están sujetas a procesos de concertación. En la medida en que la producción cultural se industrializa, las decisiones en esta área se asimilan a resoluciones económicas. Así es como la creciente oferta educativa virtual y las decisiones empresarias sobre software educativo, por ejemplo, se van alejando cada vez más de los procesos de concertación de tipo nacional.

En el caso de algunos países de América Latina, es preciso recordar que la globalización fue concomitante con la superación del autoritarismo y con el retorno al Estado de Derecho. Si bien la heterogeneidad de situaciones nacionales es muy grande, se puede sostener que, salvo casos especiales, los procesos de retorno a la democracia estuvieron asociados con reformas del Estado que provocaron su creciente incapacidad para responder a las demandas sociales. Con la pérdida de instrumentos para responder a las nuevas y a las tradicionales demandas, se ha extendido un sentimiento general de escepticismo acerca de la potencialidad de la democracia para resolver los problemas sociales. Uno de los indicadores más importantes de este clima es la desconfianza de la ciudadanía con respecto a las instituciones del Estado, hacia los otros actores sociales y hacia el propio sistema democrático ${ }^{10}$. La desconfian-

\footnotetext{
9 Un aporte muy importante a esta discusión puede verse en Ulrich Beck (2002): Pouvoir et contre-pouvoir à l'ère de la mondialisation, París, Aubier.

${ }^{10}$ Diversas encuestas y estudios nacionales y regionales dan cuenta de este fenómeno. Hace poco se presentó un estudio sobre la democracia en América Latina, realizado en 18 países. Según dicho estudio, el 54,7\% de los consultados preferiría un régimen autoritario si garantizara una mejora económica. Esta expectativa no tiene base empírica, ya que la experiencia de los gobiernos autoritarios no indica que hayan sido más capaces de resolver los problemas que los democráticos. Sin embargo, es importante tomar
} 
za, además, ha alcanzado a las instituciones y a los actores sociales responsables de la transmisión del patrimonio cultural y de la cohesión social. En encuestas a muestras representativas de docentes de varios países se ha podido apreciar que los dirigentes políticos son el grupo social sobre el cual los docentes tienen mayores niveles de desconfianza (que alcanzan entre el $70 \%$ y el $80 \%$ en todos los casos estudiados) ${ }^{11}$. La politización es percibida como negativa en la medida en que está asociada a corrupción, a clientelismo, etc. Pero tampoco gozan de confianza otros actores sociales importantes (empresarios, dirigentes sindicales, etc.).

La falta de confianza aumenta en contextos de extrema pobreza. Casos como los de Bolivia y Haití son una muestra de situaciones en las que el acuerdo es imprescindible para salir de la situación de crisis, y en los que es casi imposible lograr convenios mínimos para impulsar procesos concretos de acción. En tal sentido, la experiencia de esos países permite ver la complejidad que tiene la construcción de procesos de concertación en situaciones de emergencia social. Desde la perspectiva de las condiciones objetivas en contextos de pobreza, todas las demandas son urgentes y nadie acepta ser postergado. Los acuerdos son muy difíciles incluso entre sectores de la población que viven en condiciones de pobreza. Los conflictos de «pobres contra pobres» constituyen un fenómeno cada más frecuente en estos escenarios, donde la escasez de recursos provoca grandes pugnas entre sectores que comparten la condición de desfavorecidos. Pero en estos contextos también es preciso incorporar al análisis la dimensión subjetiva expresada a través de la desconfianza hacia el otro y hacia las conductas asociadas a los procesos de desarrollo social y político: asumir riesgos,

en cuenta este dato para asumir que el reto principal que deben enfrentar los pactos educativos es superar la mera formulación retórica para traducirse en compromisos para la acción. Ver PNUD (2004): La democracia en América Latina. Hacia una democracia de ciudadanas y ciudadanos.

${ }^{11}$ EI IIPE-UNESCO-Buenos Aires efectuó entre los años 2000 y 2001 una encuesta a muestras representativas de maestros y profesores de cuatro países: Argentina, Brasil, Perú y Uruguay. El grupo social que registró mayor nivel de desconfianza entre los docentes de dichos países fue el de «los políticos»: Argentina, 87\%; Brasil, 85\%; Perú, $76 \%$, y Uruguay, 67\%. En Argentina y Perú siguen en el ranking de mayor desconfianza los funcionarios públicos ( $84 \%$ y $62 \%$, respectivamente), los sindicalistas (sólo en el caso de Argentina. el $81 \%$ ) y los magistrados (65\% y $67 \%$ ). En el otro extremo se observa que los docentes confían masivamente en ellos mismos y después en los periodistas y en los sacerdotes. 
enfrentar la incertidumbre, etc. La desconfianza es producto de experiencias vividas por los actores sociales, que se simbolizan mediante determinadas representaciones, estereotipos y prejuicios. Por esa razón, la subjetividad ha comenzado a ser considerada como una dimensión del comportamiento social y político que debe ser tenida en cuenta para el diseño de dispositivos de procesos de concertación ${ }^{12}$.

El proceso de globalización redefine el significado que asume el ámbito local, comunitario, y todas las dimensiones asociadas a dicho espacio de participación. También sobre el tema la literatura es muy abundante, y existen evidencias que muestran que el ámbito local puede ser un espacio donde se apoyan los fundamentalismos autoritarios de carácter comunitarista, y, a la inversa, escenarios donde la concertación, las alianzas y los pactos pueden tener mayores posibilidades fácticas. En este último sentido, es posible evocar los ejemplos de pactos y de diálogos regionales de educación, así como algunas experiencias de proyectos educativos basados en la idea de comunidad de aprendizaje o de padrinazgo de escuelas por parte de empresas o de otras instituciones.

Los pactos educativos de nivel local se han visto estimulados por varias razones. Desde el punto de vista administrativo, esos pactos han adquirido relevancia en el marco de los procesos de descentralización, que en algunos casos transfirieron responsabilidades a los municipios. La experiencia más interesante en Latinoamérica es la de los «Diálogos ciudadanos por la calidad de la educación», que se llevan a cabo en Chile impulsados por la administración central ${ }^{13}$. Pero otra fuente que justifica los pactos locales es el carácter integral de las estrategias destinadas a impulsar el desarrollo comunitario. En muchos lugares se advierte la necesidad de diseñar planes estratégicos de desarrollo local o planes integrales de acción que promuevan actividades coordinadas entre distintos sectores de la administración (educación, salud, empleo, vivienda, etc.), y entre diferentes actores sociales (docentes, empresa-

12 Un análisis muy interesante de la situación de Bolivia con respecto a las condiciones subjetivas para enfrentar los procesos de desarrollo puede verse en PNUD (2004): Interculturalismo y globalización: la Bolivia posible. Informe de Desarrollo Humano 2004. La Paz.

${ }^{13}$ En estos diálogos participan profesores, alumnos, académicos, representantes del gobierno regional, padres y madres de los alumnos. Los diálogos se desarrollan en dos modalidades: presencial y vía Internet. Ya se han realizado cinco encuentros regionales presenciales, y el diálogo vía Internet se lleva a cabo a través del sitio web http:// dialogo.minenuc.cl. 
rios, Iglesia, gobierno local, etc.) $)^{14}$. Sobre la asociación entre empresas y escuelas hay abundante literatura y experiencias diversas, tanto en los países centrales como en la periferia. Hace ya varios años la OCDE publicó un libro sobre experiencias de acuerdos entre escuelas y empresas ${ }^{15}$. En él se pueden apreciar las principales motivaciones para los acuerdos entre empresas e instituciones educativas, y los límites de tales acuerdos. En primer lugar, las empresas están más dispuestas a establecer acuerdos con instituciones que con los sistemas educativos globales. En segundo lugar, hay una diferencia importante con respecto al manejo de la dimensión temporal: mientras las escuelas y los educadores tienen una dimensión de largo plazo, los empresarios pretenden resultados inmediatos.

Las condiciones del nuevo capitalismo crean situaciones en las que hay que decidir sobre cuestiones de importancia crucial para el destino personal y social, y en las que esas decisiones exigen un grado muy alto de reflexividad. Incluir o excluir, manipular genéticamente o no a las futuras generaciones, proteger o no el medio ambiente, son algunas de las opciones sobre las cuales los ciudadanos están (o deberían estar) llamados a decidir. Todas estas cuestiones ponen en juego nuestros valores, pero también nuestros conocimientos e informaciones. Las decisiones están asociadas a valores de solidaridad y de responsabilidad con el otro, así como al manejo de conocimientos científico-técnicos que nos brinden el máximo de seguridad acerca de las consecuencias de nuestras decisiones. Giddens, Beck y otros sociólogos han aludido al carácter reflexivo que tiene hoy el comportamiento ciudadano, en oposición al determinado por las fuentes tradicionales de confianza. Según sus análisis, se ha producido una significativa erosión de los cuatro ámbitos de confianza de las culturas premodernas: el sistema de parentesco, la comunidad local, la cosmología religiosa y la tradición. En las sociedades y culturas modernas el comportamiento ciudadano se basa mucho más en el conocimiento y en la información, pero la característica principal de esas fuentes de comportamiento es la de que no garantizan certidumbre. Al contrario, la validez del conocimiento es, por definición, cambiante y transitoria. En consecuencia, la confianza y la fiabilidad son mucho más difíciles de obtener. A su vez, varios analistas de los actuales procesos de cambio han señalado que las características

${ }^{14}$ N.Neirotti y M. Poggi (2004): Alianzas e innovaciones en proyectos de desanolloeducatio bcal, Buenos Aires, IIPE-UNESCO.

${ }^{15}$ CERI (1995): Schools and Business: a new Partnership, París, OCDE. 
del nuevo capitalismo son la concentración en el presente, la ausencia de sentido, y el carácter efímero de todos los vínculos sociales. Sobre eso Alain Minc ${ }^{16}$, a comienzos de los años 90 , sugirió que el nuevo capitalismo se asociaba a una nueva Edad Media, donde había ausencia de sistemas organizados, de todo centro articulador, y donde "las solidaridades serán fluidas y evanescentes». Agrega: una sociedad sin actores estructurados, sin lealtades firmes, en una estructura social "caótica», fluida, que hace imposible el consenso. La gestión, el «buen» gobierno, estarían ahora basados más en la imaginación y en el riesgo que en la confianza y la cohesión. Más allá de la vaguedad de este planteo, el punto principal que hay que retener es el de la falta de estabilidad en los acuerdos. Un grado muy bajo de estabilidad crearía una situación de caos social, mientras que un acuerdo de largo plazo sería poco sustentable por el dinamismo de la situación social. Tal panorama adquiere significados específicos en contextos de mucha desigualdad, no sólo social sino educativa. En un marco de alta reflexividad, los no-educados ocupan un lugar diferente al que tenían en sociedades en las que el comportamiento ciudadano se regulaba por alguno de los factores tradicionales. En dichos contextos la erosión de los factores tradicionales no está acompañada por el dominio de la información y del conocimiento. Se destruyen los factores tradicionales pero no aparecen los nuevos, con lo cual se exacerban los riesgos de clientelismo, de manipulación o de despotismo ilustrado. Así, sabemos que los actuales contextos de pobreza se diferencian de los tradicionales por la ruptura de los vínculos de cohesión y de confianza, y por la pérdida de capacidad para definir proyectos y para expresar demandas. Existe tendencia a vivir al día, lo que genera escasas posibilidades de participar en procesos de concertación de políticas públicas.

En síntesis, los pactos son necesarios para enfrentar la dinámica neoliberal o fundamentalista, pero, al mismo tiempo, son más exigentes en términos de articulación entre saber experto y saber lego, en los de superación de visiones particularistas, y en los de significación social de las cuestiones sobre las que los ciudadanos son convocados a pactar. Sin caer en un exceso de voluntarismo, parece importante señalar algunas características del rol del Estado en estos procesos, tanto desde el punto de vista del contenido de su acción como desde la representación de los diferentes intereses y sectores sociales.

${ }^{16}$ Alain Minc (1994): La nueva Edad Media, Madrid, Temas de Hoy. Sobre la concentración en el presente, es importante ver el análisis de Zaki Laïdi (2000): Le sacre du présent, París, Flammarion. 
Con respecto al contenido, hay dos aspectos importantes. EI primero se refiere a la responsabilidad de convocar a los diferentes actores a que negocien, a que dialoguen, a que concierten. Hay muchos ejemplos de procesos de concertación convocados y coordinados por ONG, por agencias internacionales, por la Iglesia o por una combinación de ellas. Si eso sucede es porque se ha producido una crisis profunda en la sociedad y en el aparato del Estado, pues éste es percibido como ausente o como representante de un determinado sector y no de los intereses generales. En el segundo, el Estado debe manejar la tensión que existe entre los procesos de concertación y las tomas de decisiones. La negociación no puede ser un justificativo para la inacción. Con respecto a la representación, si bien el Estado asume los intereses generales, su responsabilidad principal es representar a los excluidos. Sólo el Estado puede hablar por los que están afuera, por los no representados mediante organismos corporativos. Como sabemos, la concertación a través de organizaciones se corporativiza rápido, y los que no están organizados no participan. El ejemplo de los congresos pedagógicos es muy ilustrativo. A poco de avanzar en el proceso de discusión, los congresos se han ido corporativizando y sólo participan los que están organizados: los sindicatos, los partidos políticos, las iglesias, los empresarios, pero no los padres y mucho menos los padres de los sectores más pobres.

Tras este análisis corresponde preguntarse si, en efecto, los pactos educativos son posibles. Aunque pueda parecer muy voluntarista, el argumento final de nuestro análisis consiste en reconocer que, si algo es considerado socialmente necesario, tiene que ser posible. Lo de necesario y posible está asociado a un proyecto social y político. El pacto no es un instrumento válido en sí mismo, sino que constituye un procedimiento consistente con un proyecto social basado en la idea de construir una sociedad equitativa y dinámica. En tal contexto, concertar políticas educativas es parte de un proceso más general de fortalecimiento de la ciudadanía y de construcción de un orden político democrático. Desde esa perspectiva filosófico-social, los procesos de concertación democrática son una forma de ejercicio de la solidaridad consciente y reflexiva que exigen las nuevas estructuras sociales. Mientras en el capitalismo industrial existía lo que se concebía como solidaridad orgánica, es decir, una solidaridad semejante a la que existe entre las diferentes partes de un organismo donde no hay una decisión voluntaria de ser solidario, en el nuevo capitalismo, en cambio, los niveles de solidaridad orgánica disminuyen, y para vivir juntos será necesario querer 
hacerlo, adherirse a un proyecto político que se proponga lograr la inclusión de los excluidos, y garantizar igualdad de oportunidades a todos. La solidaridad reflexiva exige un intenso sentido de pertenencia colectiva, a partir del cual es posible aceptar la idea de la redistribución directa de los bienes. En este sentido, la educación juega un doble papel: es objeto de redistribución, y es el instrumento para formar los valores de solidaridad que permitan tomar la decisión de redistribuir. 


\title{
Contactar
}

Revista lberoamericana de Educación

\author{
Principal OEI
}

